\title{
L'exotisme dans les cinémas parisiens entre les deux guerres
}

\author{
Shahram Abadie
}

Lieux d'illusions par définition - illusion de mouvement sur l'écran, illusion de réalité des événements du film, etc. - les cinémas ont dû, dès l'origine, vendre des rêves pour prospérer. Les exploitants du cinématographe avaient très tôt compris cette condition essentielle du succès ; en témoigne la dénomination même des premiers établissements : Alhambra, Louxor, Tivoli, Capitole, etc. Rares sont, en effet, les grandes villes du monde qui n'aient eu des salles baptisées de tels noms.

L'astuce d'attirer le public par l'évocation des lieux mythiques de civilisations d'ailleurs s'est également, peu ou prou, traduite sur le plan architectural. Ainsi, des années 1910 à la seconde guerre mondiale, sont apparues en Europe comme aux États-Unis, des salles dont le décor et/ou la façade étaient inspirés, voire calqués sur des monuments - temples, mosquées, palais, etc. - de pays lointains ou de civilisations anciennes'. Certains auteurs ont vu dans ce phénomène une tendance générale au point de considérer l'exotisme comme une phase importante dans l'évolution de l'architecture cinématographique et un style typique des premiers " vrais " cinémas (Heathcote, 2001).

Le présent article vise à interroger cette thèse en étudiant trois exemples parisiens de ce type : le Louxor (1921), le cinéma Sèvres (1922) et le Rex (1932). Il s'agit d'examiner les origines, l'ampleur et les protagonistes de cet exotisme architectural des cinémas dans le contexte socioculturel, économique et artistique de l'entre-deux-guerres. L'objectif est de comprendre dans quelle mesure les campagnes de fouilles archéologiques, les expositions coloniales et l'éclectisme architectural avaient préparé, depuis la fin du XIXe siècle, un terreau sur lequel a pu s'épanouir cette tendance dans l'architecture des cinémas. Dans cette perspective, nous nous intéresserons autant, sinon davantage aux parcours des maîtres d'œuvre et d'ouvrage, et aux éventuels échanges et influences internationales, qu'à l'analyse architecturale et stylistique des salles elles-mêmes. L'examen des publications spécialisées de l'époque permettra, enfin, d'aborder la question de la réception et du devenir de cette architecture exotique.

Cette investigation s'appuie essentiellement sur nos recherches doctorales (Abadie, 2012) qui ont

1. Pour en donner quelques exemples en plus de ceux de Paris que nous analyserons, on peut citer à Londres, Regal (1928), Astoria et Palace (1929); à Berlin, Cines (1913), Filmpalast Puhlmann et Ufa-Palast Königsstadt (1925), et aux Etats-Unis, Graumans Egyptian et Mayan Theatre (1922) ainsi que les salles atmosphériques réalisées par John Eberson dans les années 1920. exploité, entre autres, les permis de construire des cinémas aux archives de la ville de Paris ainsi que les sources imprimées (revues spécialisées, recueils et manuels) parues dans les années 1920-1930. Ce travail avait permis d'établir un catalogue d'environ 200 cinémas parisiens édifiés entre 1907 et 1939. Les trois exemples sélectionnés pour la présente étude sont tirés de cet ensemble, puisqu'ils représentent les seules réalisations d'une inspiration exotique marquante à Paris pendant l'entre-deux-guerres, voire durant tout le XXe siècle. Une quatrième étude de cas aurait pu être le cinéma la Pagode (1931), mais nous ne l'avons pas retenue, car n'ayant pas été construite dès l'origine comme une salle de cinéma. Cependant, l'affectation d'un tel édifice japonisant au cinéma à cette date est en soi révélatrice d'un penchant pour l'exotisme dans l'architecture des salles.

Nous essayons, ici, de croiser les résultats de nos propres recherches avec des travaux monographiques (Humbert \&t Pumain, 2013 ; Minnaert, 2002) et des ouvrages permettant de contextualiser 


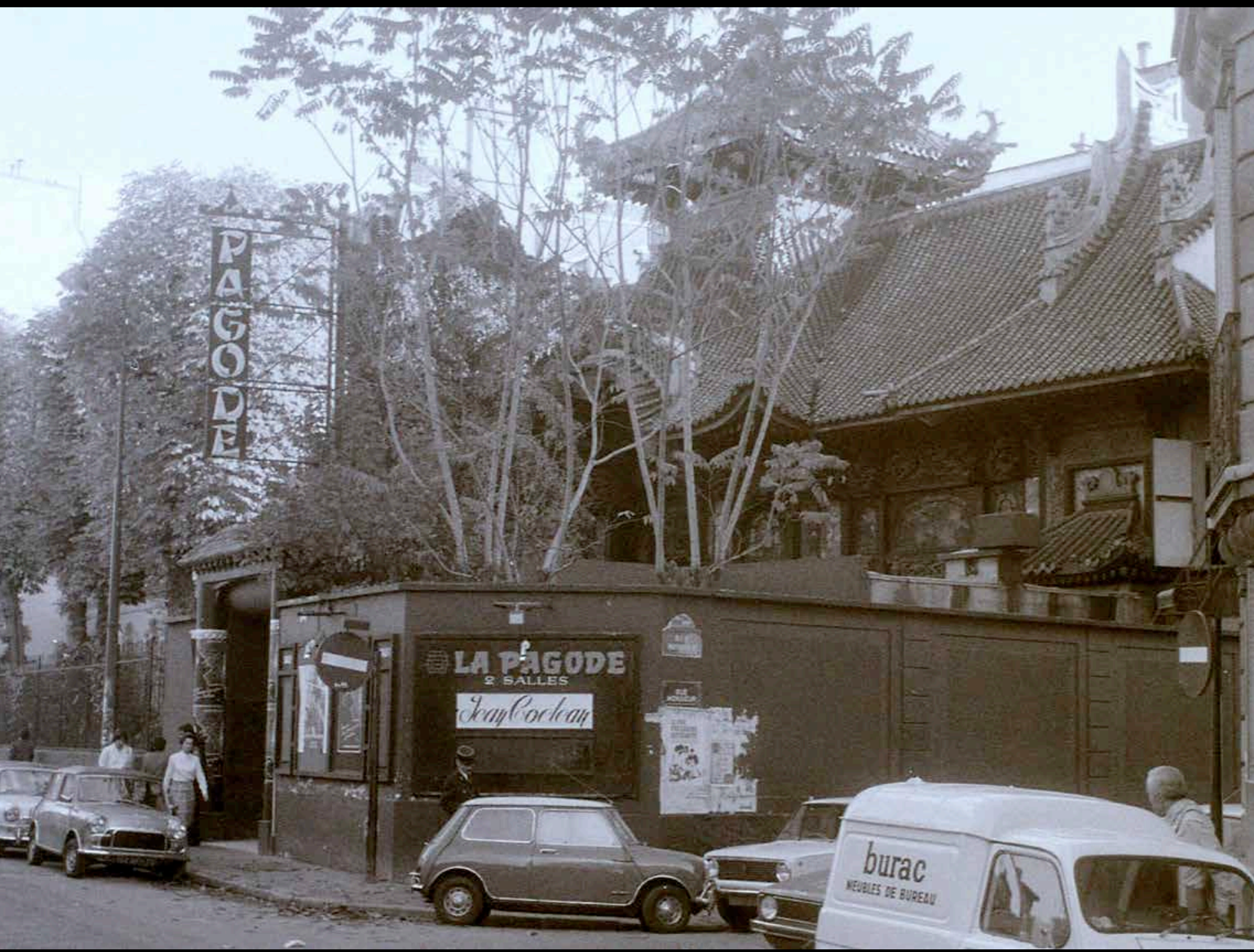

Vue de la façade $d u$ cinéma La Pagode à Paris (Photo: Lars Olsson, 1977). 


\title{
L'exotisme dans les cinémas parisiens entre les deux guerres
}

\author{
Exotismo en los cinemas parisinos del periodo de entreguerras \\ Exoticism in Parisian Movie Theaters between the Two World Wars
}

\author{
Shahram Abadie \\ ORCID : https://orcid.org/0000-0002-4735-9980 \\ chahramh@gmail.com \\ Docteur en Architecture: Université de Strasbourg. Magister en Histoire culturelle et sociale de l'architecture: UVSQ \\ - ENSA Versailles. Profesor en la École Nationale Supérieure d'Architecture de Clermont-Ferrand.
}

Résumé

Cet article interroge, à travers l'étude du cas parisien, l'idée reçue selon laquelle l'exotisme aurait marqué une phase importante de l'architecture cinématographique pendant l'entre-deux-guerres. Il s'agit de mettre en lumière les raisons d'être et l'ampleur de cette tendance en analysant le parcours des architectes et des commanditaires de cinémas ainsi que leur architecture dans le contexte des échanges internationaux avant de terminer par la réception et le devenir de ces exemples d'exotisme. La méthode consiste à croiser nos recherches doctorales avec des travaux sur l'exotisme et l'orientalisme dans l'art occidental. Cette synthèse a montré que le recours aux motifs exotiques dans les salles parisiennes est relativement précoce, mais ce mode décoratif reste marginal par rapport à la tendance Art déco. Cependant, bien que leurs premiers exploitants aient tous fait faillite, la singularité de ces cinémas a été le principal garant de leur survie et préservation comme patrimoine architectural et cinématographique.

Mots-clés: architecture, salles de cinéma, façades, architecture intérieure, éclectisme, modèles architecturaux

Resumen

A través del estudio del caso parisino, se analiza la idea según la cual un fenómeno de exotismo habría marcado una fase importante en la producción de la arquitectura para la exhibición cinematográfica durante el período de entreguerras. El presente artículo indaga acerca de las razones y el alcance de dicha tendencia, apoyándonos tanto en el estudio de las trayectorias y relaciones entre arquitectos y propietarios de salas de cine, como en el estudio de la arquitectura interior y exterior de estas, producida en un contexto de intercambios internacionales, y sin olvidar la importancia de la difusión y recepción de la cual fueron objeto. Para ello, se ponen de relieve conexiones entre nuestra investigación doctoral, con los resultados de otras investigaciones que han abordado cuestiones relacionadas con el exotismo y el orientalismo en el arte occidental. Así, se muestra como el uso de motivos exóticos de diversos orígenes fue relativamente precoz en los cinemas parisinos, aunque finalmente ello resultara marginal en comparación al uso dado a elementos propios del Art Deco. Sin embargo, la singularidad de dicho exotismo aplicado a las arquitecturas para la exhibición cinematográfica, ha sido por otro lado, una de las principales razones de su supervivencia y eventualmente de su preservación como patrimonio arquitectónico y cinematográfico.

Palabras clave: arquitectura, salas de cine, fachadas, arquitectura interior, eclecticismo, modelos arquitectónicos

Recibido : 1 de mayo de 2016

Aprobado : 1 de febrero de2017

Disponible en línea: 18 de junio de 2018
Abstract

This article examines, through the study of the Parisian case, the idea according to which the exoticism would have marked an important phase of the cinema architecture during the interwar period. The aim is to highlight the reasons and the scope of this trend by analyzing the history of the architects and sponsors of the cinema and its architecture in the context of international exchanges. The paper concludes with the issue of the reception and the destiny of these examples of exoticism. The methodology consists of crossing our doctoral research with existing work on exoticism and orientalism in Western art. This synthesis has shown that the use of exotic motifs in Parisian cinemas is relatively early, but this decorative mode remains marginal compared to the Art Deco trend. However, although all their first owners have failed, the uniqueness of these cinemas has guaranteed their survival and preservation as an architectural and cinematographic heritage.

Keywords: architecture, movie theaters, facades, interior architecture, eclecticism, architectural models

doi:10.11144/Javeriana.apc31-1.edcp 
notre thème d'étude (Epron, 1997 ; Oulebsir \&t Volait, 2009 ; Humbert, Pantazzi \&t Ziegler, 1994). La démarche consiste à analyser, d'abord, la nature et les caractéristiques des éléments exotiques composant l'architecture et le décor de chacune des études de cas à partir des plans d'origine et des documents iconographiques divers, puis de mettre en parallèle ces constats avec les origines culturelles et les parcours professionnels des commanditaires et des architectes tout en étudiant le contexte d'élaboration des projets afin d'éclairer, autant que possible, les motifs et les raisons des choix stylistiques. Nous tenterons, par ailleurs, une mise en perspective de ces exemples d'exotisme parisiens par rapport aux réalisations contemporaines, reproduites dans la littérature spécialisée ou diffusées dans les sources imprimées.

\section{Le décor dans les salles obscures}

Les premiers cinémas construits en dur à Paris, entre 1907 et 1909, s'implantent majoritairement dans les quartiers périphériques et populaires, misant sur une clientèle modeste composée de familles ouvrières. lls sont le plus souvent des hangars aménagés à l'économie en salles de spectacle, partant le décor y est maigre, voire inexistant, se limitant à quelques touches d'ornement : une bande de guirlandes et des encadrements moulurés sur les parois latérales, parfois autour de l'écran, et plus rarement, autour de l'oculus de la cabine de projection (fig. 1). C'est à compter de 1910, avec un regain d'intérêt de la bourgeoisie pour le cinéma et la prolifération des établissements dans les quartiers cossus que la décoration des salles prend une importance grandissante, sans doute afin de reproduire l'ambiance familière des théâtres et opéras aux yeux du nouveau public de marque. Ainsi les cinémas s'enrichissent-ils en ornement, puisant dans le répertoire des formes et motifs classiques ou baroques : pilastres, corniches et frontons, cartouches, volutes et mascarons. Quelques réalisations Art nouveau ${ }^{2}$ se manifestent aussi çà et là, à l'instar du cinéma Artistic Pathé conçu par l'architecte Marcel Oudin en 1912. C'est essentiellement dans la continuité de ces trois tendances décoratives que la majorité des cinémas sont édifiés après la Grande Guerre. Parmi les nouveaux établissements des années 1919-1921, un tiers se caractérise, en effet, par un décor sobre et ponctuel.

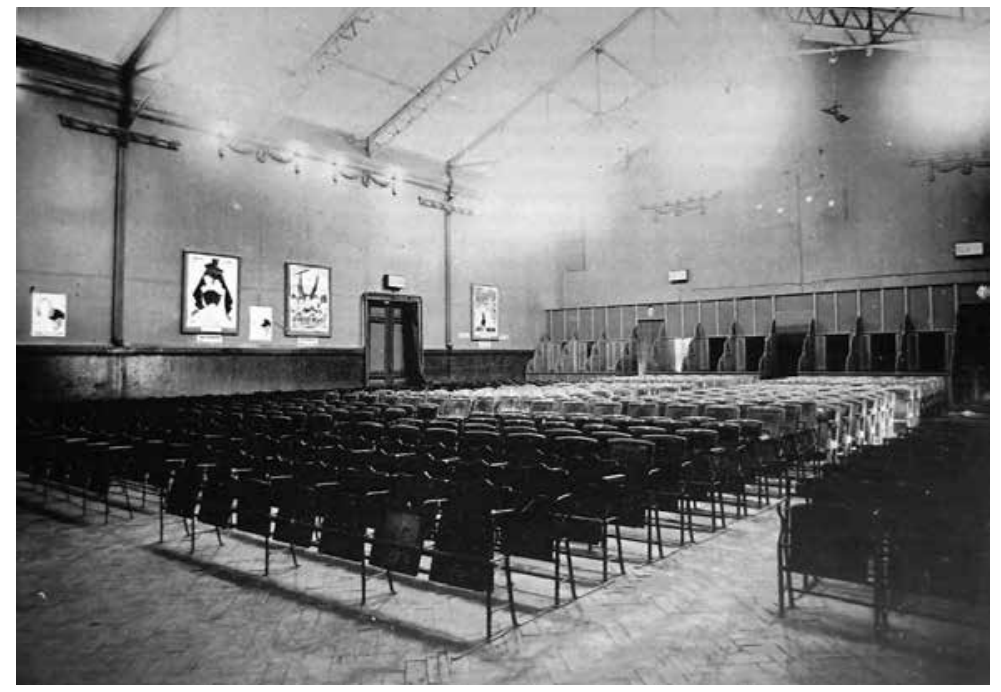

Contrairement à l'avant-guerre où l'absence de décor rimait avec quartiers populaires, ceux-ci sont répartis équitablement à travers la ville, et pour la plupart réalisés par des architectes peu connus. Mais on y trouve aussi des constructions de maîtres réputés pour leur inspiration Art nouveau, comme Auguste Bluysen et Marcel Oudin. Par conséquent, la sobriété décorative semble, dans ces cas, le choix des commanditaires, souvent faute de moyens. À l'opposé de ces salles plus ou moins austères, il en est d'autres richement décorées dans un style néoclassique, évoquant théâtres et opéras du XIXe siècle. Généralement établies dans des quartiers centraux ou aisés de Paris, elles comptent souvent plus d'un millier de places. Le Regina Aubert Palace (fig. 2) et le Palais des Glaces en représentent deux exemples réalisés respectivement par les architectes Henri Belloc et Constant Lefranc, tous deux diplômés de l'École des beaux-arts.

Le décor classicisant n'est pas pour autant l'apanage des anciens élèves des Beaux-Arts ; Émile Thion, qui n'a pas été formé à cette école, opte également pour ce mode décoratif dans la conception du Grand Cinéma de l'avenue Bosquet, même s'il sera plutôt connu pour sa façade Art déco du Cinéma Opéra de Reims construit en 1923. À cette époque, à Paris aussi, un cinéma sur trois est décoré dans un mélange d'Art nouveau tardif et d'Art déco avant la lettre. Bien que réalisés pour des commanditaires différents, la plupart de ces salles sont signées par deux architectes: Marcel Oudin et Eugène Vergnes, auteurs respectivement et entre autres du cinéma Madeleine (1920) caractérisé par des motifs géométriques mis en valeur sous un éclairage ad hoc (fig. 3), et du
Fig. 1 :

Cinéma Pathé Bagnolet, Paris 20 arrond., Georges Malo arch., 1907. Vue de la salle vers 1930 (Source :Meusy, 2002 :200).

2. Art nouveau dans le sens défini par François Loyer, comme un mouvement architectural développé à compter de 1907-1908 en France et surtout à Paris, se caractérisant par l'abandon d'excès et d'exubérance for melle de l'art floral, qualifié tour à tour d'" Art nouveau assagi " et " adouci ", et d'" école typiquement parisienne de l'Art nouveau tardif " (Russell, 1982 : 134). 
3. Pour plus d'illustrations et une description détaillée de la salle voir (Humbert \& Pumain, $2013: 36$ et $81-87$ ).

Fig. 2 : Régina Aubert Palace, Paris 6 arrond., Henri Belloc arch., 1919. Coupe transversale d'origine révélant le décor côté écran (Source : Archives de Paris : Vo11 3002). cinéma Danton (1921) orné de grandes fresques pittoresques.

C'est en marge de ces trois tendances principales que l'on relève quelques recherches d'originalité dans la décoration des cinémas, par exemple au Louxor, un grand cinéma d'environ 1300 places, dessiné par l'architecte Henri Zipcy (1873-1950) pour un exploitant apparemment novice, Henri Silberberg (1866-1921). Tout à l'image du nom de l'établissement, le décor et la façade sont conçus dans un esprit ostentatoire d'égyptomanie (fig. 4). Les parois de la salle sont peintes en colonnes florales, balisées de pilastre feint, le tout surmonté d'une frise de lotus et de papyrus, de procession d'Égyptiennes et de têtes pharaoniques en relief. La même frise couronne le cadre ornementé et doré de l'écran. Un motif symbolique, le disque ailé, formé d'une paire d'ailes grand ouvertes et d'un disque solaire flanqué de cobras, se répète sur les garde-corps et le cadre de l'écran, et au plafond, des lignées de hiéroglyphes couvrent les poutres ; l'ensemble créant l'ambiance fantaisiste, presque mystérieuse d'un temple, voire d'un tombeau égyptien antique 3 . La plupart des motifs employés dans la décoration intérieure sont également repris en façade. La composition générale de cette dernière, la volumétrie et les proportions sont conçues de manière

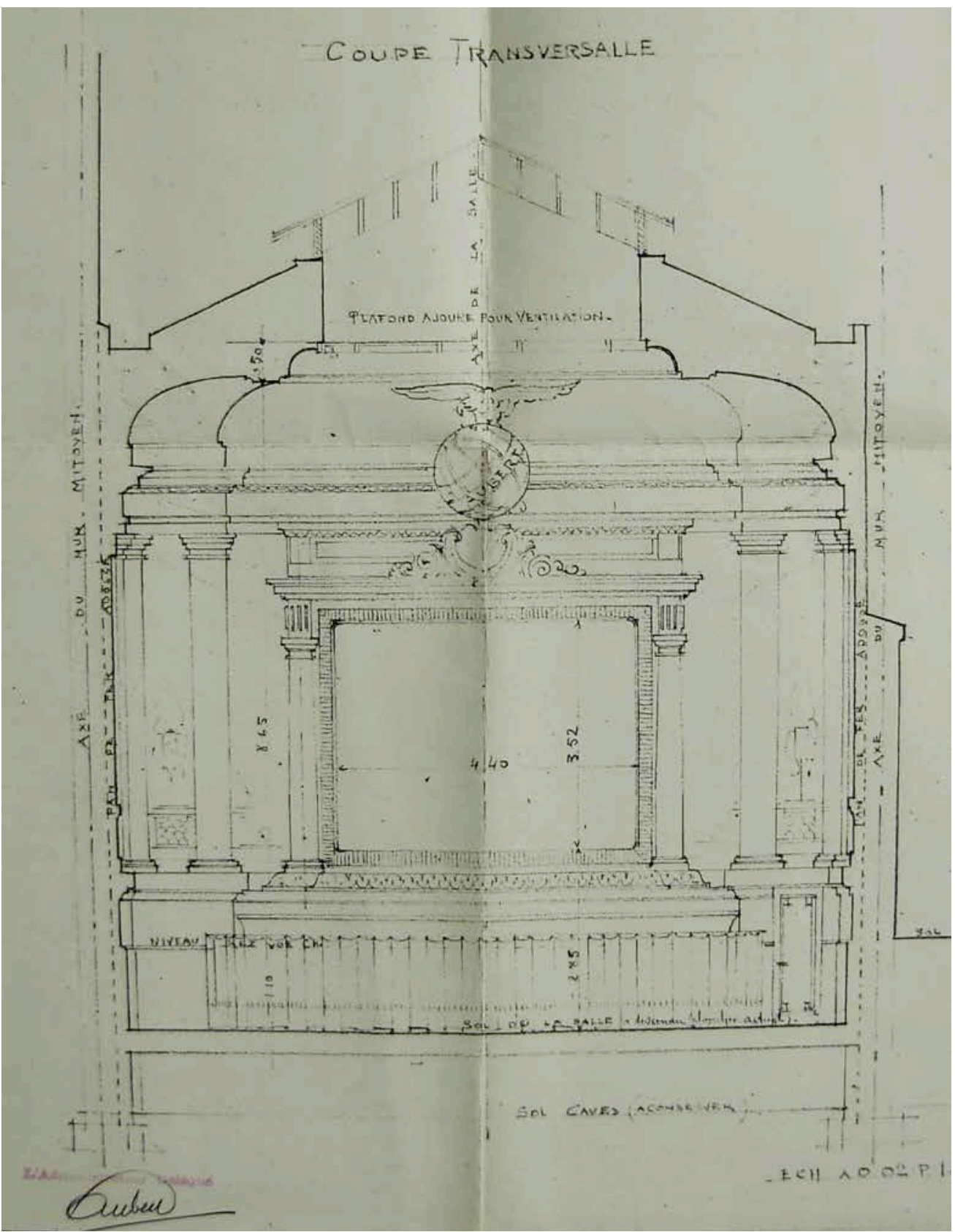


à reproduire l'aspect d'un vrai temple égyptien, accentué par des colonnes monostyles à chapiteaux papyriformes et des murailles aveugles (fig. 5$)^{4}$.

Quelles sont l'origine et la motivation de cet exemple singulier d'égyptomanie dans un cinéma parisien ? S'emparer de noms exotiques pour singulariser son établissement et intriguer le public n'est, certes, pas une astuce sans précédent ; les deux cinémas baptisés Alhambra, dans le 19e et le 20e arrondissement, datant de 1907 et de 1920, l'attestent. Mais cela n'a pas produit, jusqu'alors, d'incidence sur l'architecture et le décor de la salle, du moins à Paris et à notre connaissance.

\section{L'exemple égyptisant : le Louxor}

l'égyptomanie dans l'art européen de l'époque moderne a des racines historiques remontant au moins au XVIlle siècle, avec des moments de recrudescence au gré des évènements tels que l'Expédition d'Égypte de Bonaparte, le déchiffrement des hiéroglyphes, l'ouverture du canal de Suez et la découverte du tombeau de Toutankhamon. De plus, l'architecture a été, depuis les années 1800, l'un des domaines de prédilection de cette tradition (Humbert, Pantazzi \&t Ziegler, 1994 : 22). D’un autre côté, l'engouement pour les formes et motifs égyptiens pourrait s'expliquer dans un contexte plus large d'orientalisme architectural en France dont Pierre Pinon a cherché les fondements dans les cours d'histoire de l'architecture de Jean-Nicolas Huyot à l'École des beaux-arts entre 1823 et 1840 (Oulebsir \& Volait, 2009 : 13-26). Cette initiation aux architectures d'ailleurs a probablement pu nourrir la pratique des architectes formés dans une école qui, " par son organisation et par son principe de fonctionnement, institutionnalise l'éclectisme " durant la seconde moitié du XIXe siècle (Epron, 1997 : 80). Un des produits emblématiques de ce mode de conception architecturale est observé dans le palais de l'Égypte à l'Exposition universelle de 1900 à Paris. Réalisé par Marcel Dourgnon (18581911), architecte diplômé de l'École des beauxarts, cet édifice est un " patchwork " mêlant les styles égyptiens antique et arabisant (Humbert, 2010) (fig. 6).

Malgré son succès modeste par rapport aux " vedettes " de l'événement, ce grand pavillon a pu marquer les esprits des protagonistes du projet du Louxor, qui sont alors tous deux jeunes et à

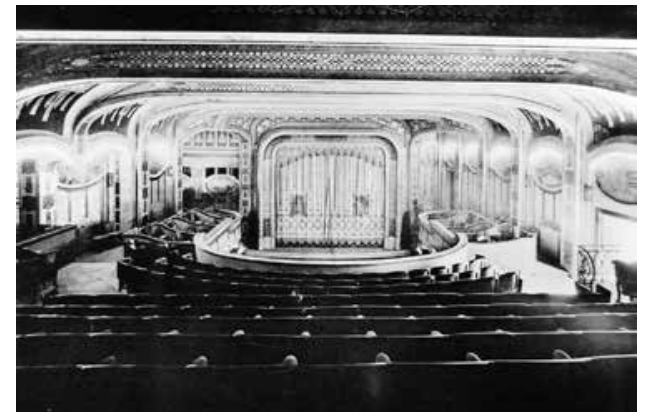

Fig. 3 :

Cinéma Madeleine, Paris 9 arrond., Marcel Oudin arch., 1920. Mise en valeur du décor par l'éclairage (d'après Vergnes, 1925).
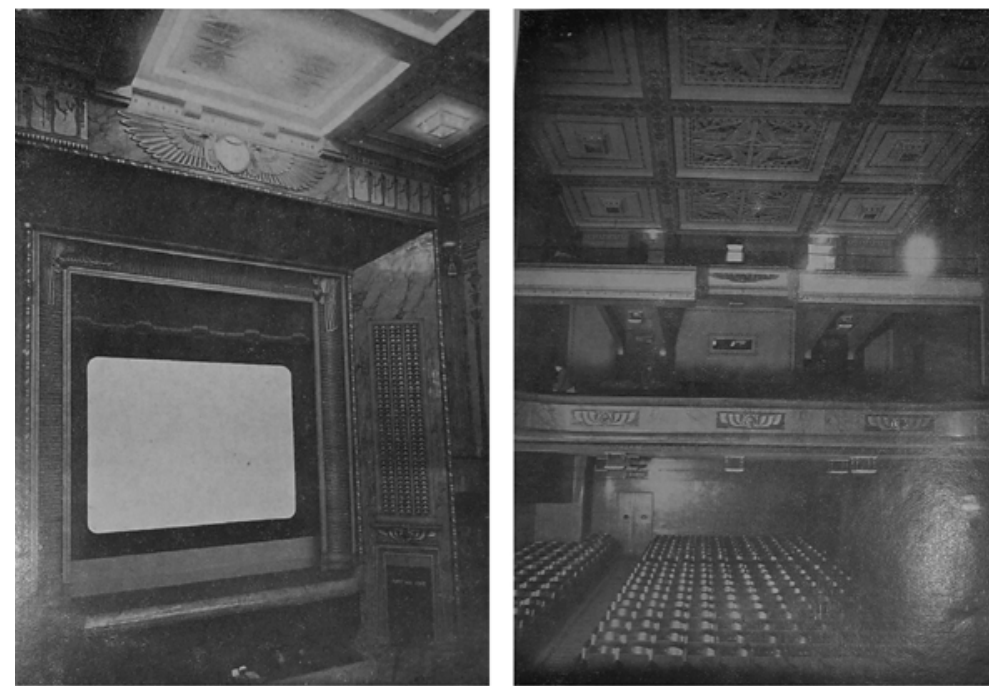

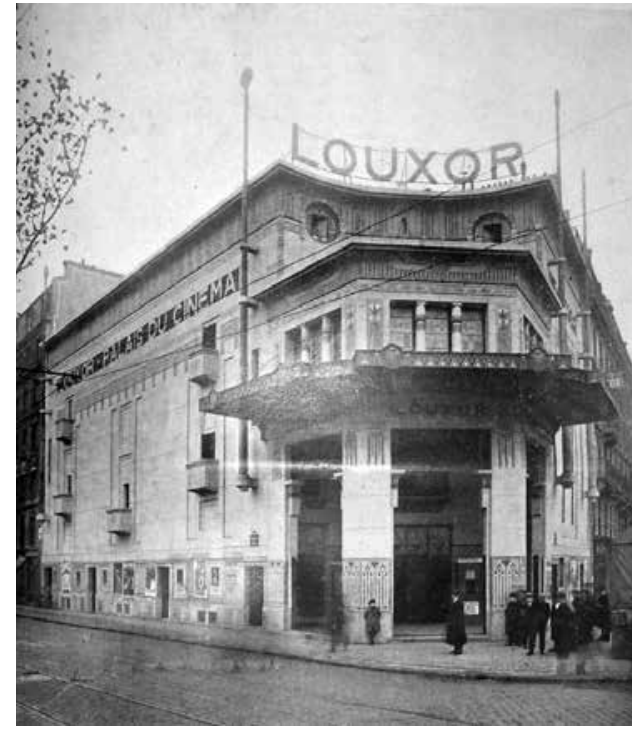

peu près trentenaires. La question est justement de savoir dans quelle mesure ceux-ci ont suivi la mode, ou fait preuve d'originalité dans la réalisation de cet établissement mythique de Paris.

Le commanditaire du Louxor, Henri Silberberg est un " Parisien " né dans une famille modeste, originaire de l'Europe de l'est, qui a émigré en France via l'Égypte, mais juste l'année d'avant la naissance d'Henri ! Avant d'entreprendre la
Fig. 4 :

Cinéma Louxor, Paris 10 arrond. Henri Zipcy arch., 1920. Décoration égyptisante de la salle (Source : $\mathrm{La}$ Construction moderne, 37e année, $\left.n^{\circ} 26\right)$.

Fig. 5 :

Cinéma Louxor, Paris 10 arrond, la façade vers 1922 (Source : La Construction moderne, 37e année, $\left.n^{\circ} 26\right)$.

4. Par ailleurs, la position de cet édifice à l'angle de deux boulevards avec sa composition à pan coupé et en gradins, sa marquise étendue et son enseigne lumineuse lui confèrent un aspect de proue de navire, un exemple de style paquebot avant la lettre. 
Fig. 6 :

Le palais de l'Égypte à

l'Exposition universelle de 1900, Paris, Marcel

Dourgnon arch. (Source : Humbert, 2010).

Fig. 7 :

Grauman's Egyptian Theatre, Los Angeles, États-Unis, Meyer \& Holler arch., 1922. Vue de l'écran (Source : Harold Allen, 1950, ${ }^{\circ}$ SAIC).

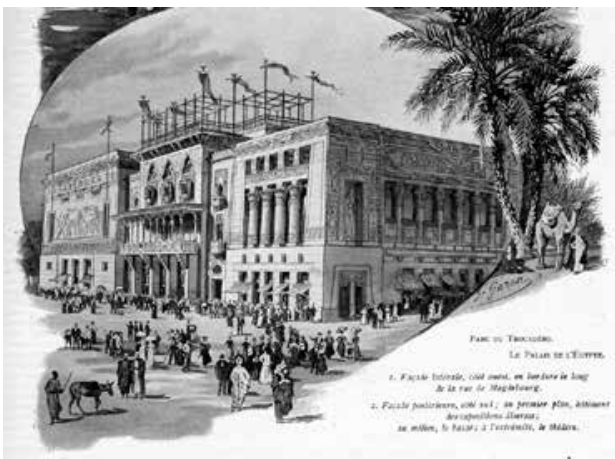

construction d'un cinéma à Paris, il s'est lancé dans plusieurs affaires dont un négoce de chaussures, la direction d'un casino et une agence de publicité, mais le succès n'est pas toujours au rendez-vous. Le parcours de cet homme d'affaires est en effet scandé de faillites dont celle de l'exploitation du Louxor dès l'inauguration, qui lui sera fatale (Humbert \& Pumain, 2013 : 30). Quoi qu'il en soit, a priori, rien dans le passé du commanditaire ne permet de supposer un quelconque attachement à l'art et à l'architecture de l'Égypte antique. Son architecte, en revanche, est d'origine arménienne, natif de la Turquie, ou de l'Empire Ottoman plus exactement, qui comprend aussi l'Égypte encore à cette époque, jusqu'à la colonisation anglaise après 1882. De famille francophone, Henri Zipcy intègre l'École des beaux-arts à ses 19 ans, pour faire des études d'architecture au sein de l'atelier Gaston Redon (1853-1921). Il est admis en première classe, au bout de cinq ans, en 1897, mais comme beaucoup d'élèves à cette époque, ne passe pas son diplôme qui n'est absolument pas obligatoire, ni nécessaire pour exercer en tant qu'architecte. En 1907, il est signalé comme " architecte à Constantinople " (Delaire, 2004 : 430). L'atelier qu'il fréquente à l'école parisienne est réputé pour son " enseignement fort peu scolaire " sous la direction de Gaston Redon qui semble " profondément désintéressé des architectures ordinaires "

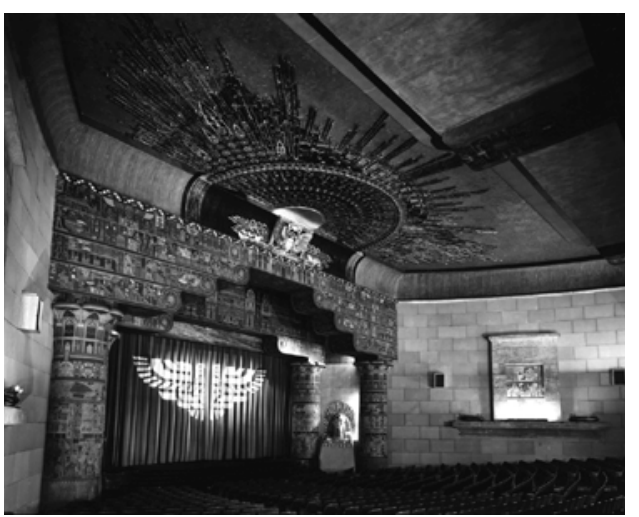

et attiré par la fantaisie (Epron, 1992 : 122). Or, cela n'aura pas empêché le maître de former des Grands Prix de Rome tels que Michel Roux-Spitz et Henri-Roger Expert dont l'œuvre se caractérise par un modernisme tempéré. Par conséquent, on ne relève guère d'indice d'un penchant orientaliste dans le parcours scolaire d'Henri Zipcy, au-delà du contexte général de l'éclectisme de l'École des beaux-arts entendu comme " la liberté de choix " des éléments de composition (Epron, 1997). Par ailleurs, l'appartenance de l'architecte du Louxor à une culture orientale ne saurait expliquer, seule, la proposition d'une telle conception égyptisante, puisque le jeune Zipcy épouse une Italo-allemande à lstanbul, pour revenir s'installer à Paris dès 1914, obtenir la nationalité française en 1928 et donner à ses enfants une éducation française " sans rien transmettre de leurs origines arméniennes ottomanes " (Humbert \&t Pumain, 2013 : 31).

Hormis les intérêts et intentions des principaux protagonistes, il semble que le projet du Louxor porte la marque des sensibilités contemporaines. D'une part, ce que l'on appelle " l'orientalisme moderne ", surtout en peinture, connaît un âge d'or au lendemain de la Grande Guerre (Peltre, 2010 : 210-214), accusant un fort intérêt pour les cultures d'ailleurs. Au sein de cet exotisme artistique, l'inspiration des formes et des motifs égyptisants se manifeste dans des domaines aussi divers que la publicité et la lithographie, les arts dramatiques et décoratifs jusqu'à l'architecture (Humbert, Pantazzi \&t Ziegler, 1994 : 508-511). D'autre part, l'Égypte islamique et plus tard antique, avait charmé les architectes français, depuis une cinquantaine d'années, en particulier suite aux premières fouilles archéologiques entamées dans les dernières décennies du XIXe siècle. La fascination pour la civilisation pharaonique est stimulée, de temps à autre, par des découvertes de sites antiques dont l'une des plus retentissantes, celle de la sépulture de Toutankhamon par le Britannique Howard Carter, en 1922. Or, cet événement marquant a eu lieu deux ans après l'élaboration du projet du Louxor, ce qui réfute une influence directe. Comme le fait remarquer Michael Pantazzi, il n'est pas facile d'évaluer l'impact de cette découverte sur les arts " en dehors d'un contexte social élargi ", car " à cette date, le répertoire des manifestations ayant trait à l'Égypte était si étendu en Occident qu'on a quelque peine à faire la distinction entre ce qui a précédé cette découverte et ce qui l'a suivie " (Humbert, Pantazzi \&t Ziegler, 1994 : 508). 
En tout cas, à ce début des années 1920, l'Antiquité pharaonique est en honneur dans les salles de cinéma, non seulement à Paris, mais aussi sur la côte ouest des États-Unis. Sid Grauman, un exploitant américain, commanditaire de plusieurs salles, ouvre en 1922 sur le Hollywood Boulevard un établissement baptisé Egyptian Theater. Édifié par les architectes Meyer et Holler, il est décoré dans un style comparable au Louxor parisien, quoique nettement plus surchargé en motifs empruntés à l’Égypte antique (fig. 7).

L'auteur de Cinema Builders inscrit cette réalisation dans un courant général d'exotisme architectural des cinémas, développé aux États-Unis dans les années 1920. 11 en cherche les prémices dans le Cines-Theatre d'Oskar Kaufmann, réalisé en 1911 à Berlin, dont le porche fut couronné d'une statue de Bouddha (Heathcote, 2001 : 16). L'auteur britannique va même jusqu'à en faire remonter la généalogie à l'Egyptian Hall de Londres, érigé en 1812 à Piccadilly, par la suite affecté brièvement aux représentations d'images animées. D'autres ont cité des réalisations et des publications américaines pendant la Grande Guerre comme lanceur de la tendance égyptisante, en particulier le manuel Modern Theatre Construction (Kinsilla, 1917) qui, distinguant les cinémas des théâtres, recommande les formes égyptiennes, entre autres, comme décoration pour les premiers car convenant le mieux " au goût du plus grand nombre " (Humbert, Pantazzi \& Ziegler, 1994 : 513). Le Louxor est souvent mentionné, notamment par Edwin Heathcote, comme preuve d'admission de cette vogue à Paris. Or, il s'agit en réalité d'un cas unique dans la capitale française voire même dans tout le pays où l'on ne connaît pas d'exemple comparable, par conséquent, ce serait exagéré d'en parler comme d'un style à la mode dans les cinémas français.

D'autre part, cette salle parisienne précède d'au moins deux ans les cinémas de style égyptien construits outre-Atlantique tels que le Grauman's Egyptian Theatre mais aussi l'Egyptian Theatre d'Ogden dans l'Utah (1924) et le Zaring's Egyptian Theatre à Indianapolis (1925) (fig. 8; fig. 9). Tout cela révèle la singularité du Louxor et la difficulté d'expliquer sa genèse dans le contexte parisien.

\section{Exotisme dans les cinémas d'Henri Sauvage}

Parmi les salles parisiennes des années 1920, seul le Cinéma Sèvres réalisé par Henri Sauvage (1873-
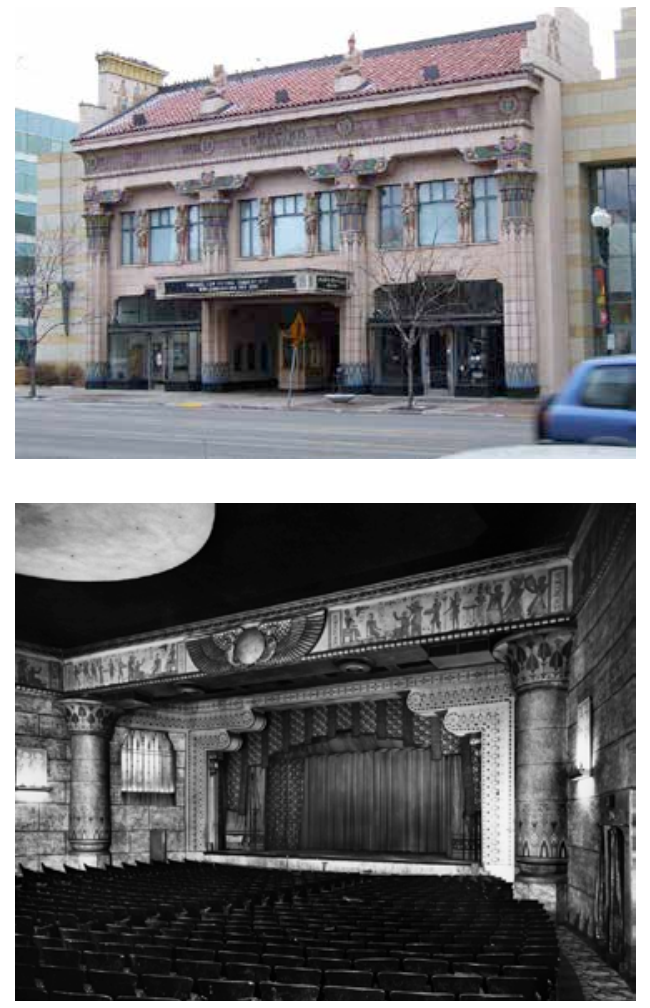

1932) présente des motifs décoratifs d'inspiration ouvertement exotique (fig. 10). Cet architecte, l'un des plus importants et prolifiques du premier tiers du XXe siècle en France, est surtout célèbre pour son concept des immeubles à gradins dans la mouvance hygiéniste. Mais en tant que designer et décorateur, il a laissé une œuvre abondante représentative des courants esthétiques successifs depuis la fin du XIXe siècle jusque dans l'entredeux-guerres : de l'Art nouveau à l'Art déco et du régionalisme au modernisme (Minnaert, 2002). $\mathrm{Au}$ lendemain de la Grande Guerre, à l'apogée de sa carrière, il fait partie des architectes sollicités par les exploitants de cinéma pour créer des salles adaptées à ce nouveau spectacle qui connaît la pleine prospérité et s'érige progressivement au rang d'un art à part entière. Cependant, les deux premières études de Sauvage pour des salles de spectacle n'atteindront pas la phase de réalisation. C'est avec ses deux projets suivants, le Gambetta Palace et le Cinéma Sèvres, qu'il entre dans le cercle des constructeurs de cinémas parisiens. Le commanditaire de la salle de la rue de Sèvres, un certain M. Soulé, est contrairement à son architecte, une figure inconnue de l'exploitation cinématographique. 11 nous est donc impossible de vérifier si il a eu un rôle dans le choix du mode décoratif de la salle. En tout cas, la décoration conçue par Henri Sauvage se compose de deux
Fig. 8 :

Egyptian Theatre,

Ogden, Utah, États-Unis, Hodgson \& McClenahan arch., 1924. La façade (Source : Joe Hall 2006).

Fig. 9 :

Zaring Egyptian Theatre, Indianapolis, États-Unis, Rubush Et Hunter arch., 1925. Décor égyptisant de la salle (Source : Harold Allen, 1953, ${ }^{\circ}$ SAIC). 
Fig. 10 : Cinéma Sèvres, Paris 7 arrond., Henri Sauvage arch., 1920. Façade ornée de masques d'Indiens et de motifs précolombiens (Source : Vergnes, 1925).

Fig. 11 : Cinéma Sèvres, Paris 7 arrond., vue de la salle avec le décor aux motifs floraux et indiens encadrant la scène (Source : Vergnes, 1925).

Fig. 12 : Mayan Theater, Los Angeles, ÉtatsUnis, Morgan, Walls \&t Clements, arch., 1922 (Source : la Library of Congress, domaine public).

5. Seul exemple qui s'en approche est le Montrouge Palace, construit en 1921 par Marcel Oudin qui fait installer, dans les angles de la salle, du côté de l'écran, deux fontaines lumineuses, créant ainsi une composition dont Eugène Vergnes admirera l'" attrait particulier et artistique " (Vergnes, 1925 : p. 13).
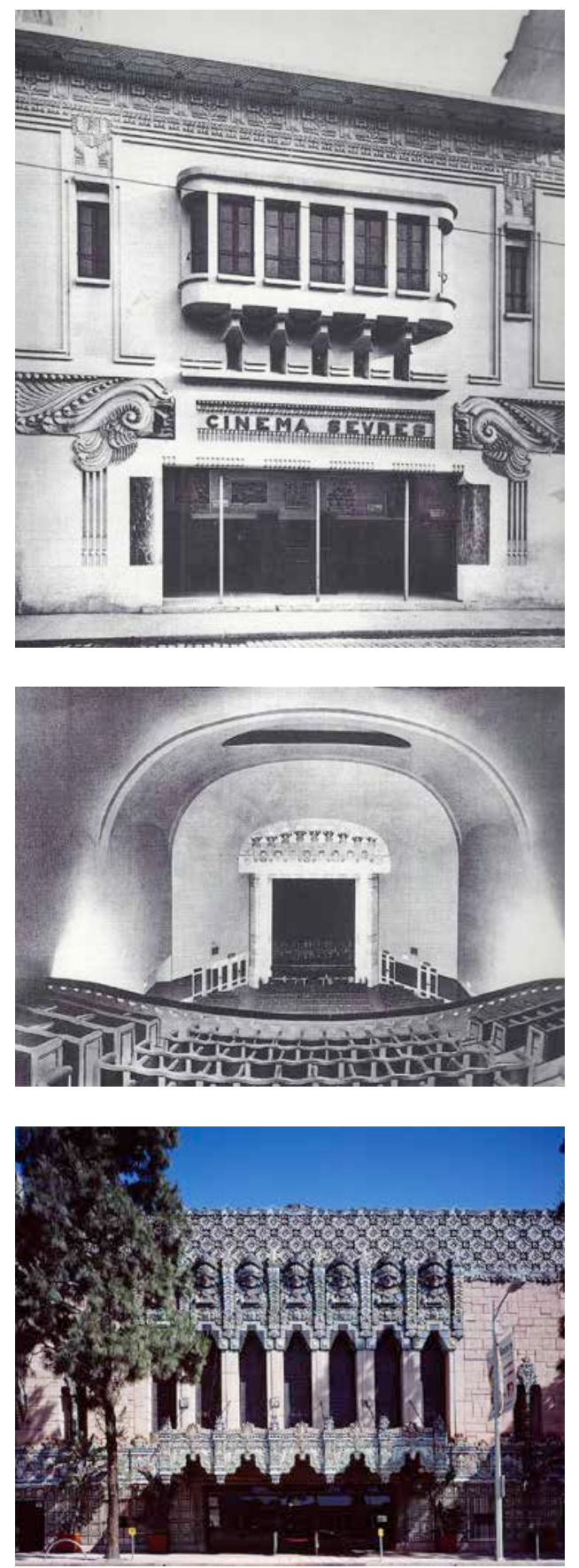

dispositifs dont l'un " matériel ", sobre et ponctuel, consiste dans un encadrement en marbre de la scène et de l'écran, couronné d'un fronton sculpté de masques et de motifs visiblement précolombiens (fig. 11). L'autre dispositif utilise la lumière comme un élément décoratif à part entière, selon un système original, et même breveté par l'architecte.

Ce " décor lumineux ", changeant et chatoyant, procède, d'après la description d'Eugène Vergnes, de la projection des images sur les murs lisses de la salle par des projecteurs électriques dissimulés dans les trois voûtes annulaires du plafond (Vergnes, 1925 : 14). Cette disposition paraît singulièrement précoce et n'aurait pas d'équivalent, à notre connaissance, parmi les cinémas contemporains ${ }^{5}$. L'assemblage de ces deux modes décoratifs si différents dans cette salle n'est pas sans poser question : d'un côté, un décor lumineux très moderne, une quasi curiosité technique, de l'autre, des motifs antiques sculptés dans le marbre ! Faudrait-il y voir l'intérêt du maître d'ouvrage pour les civilisations d'Amérique latine qui aurait amené l'architecte à faire le grand écart ? Rien ne parait moins sûr dès lors que l'on examine la façade du cinéma. En effet, de même qu'au Louxor, au Cinéma Sèvres on relève une concordance parfaite entre l'intérieur et l'extérieur. La façade, d'une composition tripartite, est marquée par un porche central surmonté d'un bow-window, en réalité l'arrière de la cabine de projection en saillie sur la façade. L'ornementation de cette dernière fait clairement écho à celle de la salle par deux masques d'Indiens réalisés en marbre et ciment de parement, de part et d'autre de l'entrée ainsi qu'une bande de motifs rappelant les figures mayas ou aztèques à la naissance de la corniche. Cette architecture fortement caractérisée par des formes exotiques ne saurait être uniquement imposée par le commanditaire. D'ailleurs, le biographe d'Henri Sauvage évoque un vraisemblable recours de l'architecte dans la conception de ce décor, à l'ouvrage d'Eugène Grasset, Méthode de composition ornementale, une des références des précurseurs de l'Art déco à cette époque (Minnaert, 2002 : 237).

Le Cinéma Sèvres semble résulter, ainsi, d'un croisement des recherches qui s'inscrivent dans un mouvement aspirant à un nouveau " style ", et des inspirations fantaisistes qui visent à identifier le cinéma au voyage et à la découverte d'ailleurs. Mais on peut s'interroger sur les raisons et l'originalité du choix des motifs précolombiens. À cet égard, il est intéressant de noter que, même dans l'inspiration des anciennes civilisations d'Amérique, l'exemple parisien devance son équivalent américain, le Mayan Theater de Los Angeles, construit en 1922 ; même si ce denier déploie un décor beaucoup plus surchargé, marqué par une exubérance de formes et des proportions titanesques (fig. 12). Par conséquent, quand bien même on admettrait la thèse d'Edwin Heathcote sur l'exotisme des cinémas des années 1920, développé et promu par les Américains, il n'en reste 
pas moins que cette tendance est de moindre importance en France et à Paris, notamment par rapport au mouvement Art déco, et en tout cas, ne procède pas de l'influence hollywoodienne puisque les salles " exotiques " parisiennes sont antérieures à leurs équivalents outre-Atlantique.

D'ailleurs, parallèlement à ces tentatives marginales d'exotisme, il faudrait souligner quelques cas singuliers de rejet d'ornementation dans les cinémas de Paris, à l'instar du Gambetta Palace, l'autre salle réalisée par Henri Sauvage. lci, les parois lisses et incurvées de la salle, délibérément dépouillées de tout ornement, conduisent le regard vers l'unique composition décorative: une arcade recouverte d'un rideau aux motifs végétaux, qui enveloppe la scène et encadre l'écran, sans doute pour le mettre à l'échelle de l'espace et y focaliser l'attention des spectateurs (fig. 13). Sur cette nouvelle façade conçue par Henri Sauvage, aucune référence aux territoires lointains. Tirant parti de l'emplacement du cinéma à l'angle de deux rues, l'architecte dessine une paroi galbée qui commande le tracé du décor. Trois bandes de motifs polychromes en relief courent sur ce mur convexe : la première, à la hauteur du " soubassement ", composée d'entrelacs de formes géométriques et florales ; la deuxième, à la naissance de " l'étage noble ", formée d'alternance d'horizontales et de petites fenêtres ; la troisième, créée par une série de marionnettes et de vitraux de grotesques. Une large corniche fait ressortir, par un jeu d'ombre, le galbe de cette façade davantage Art déco qu'exotique (fig. 14). En effet, le Louxor et le Cinéma Sèvres resteront une décennie durant les seuls exemples d'exotisme dans l'architecture cinématographique parisienne. Dans un contexte de ralentissement de construction de cinémas à partir de 1922 (Abadie, 2018, p. 129), les rares salles qui voient le jour, à l'instar du Paramount Palace (1926), témoigne de la grande influence du mode décoratif consacré par l'Exposition des arts décoratifs de 1925 à Paris (fig. 15).

\section{Le Rex : l'exotisme made in U.S.A}

C'est dans la vague de construction des grands cinémas suivant l'avènement du film parlant que l'on peut repérer l'un des derniers exemples, sinon le chant du cygne de l'exotisme dans l'architecture cinématographique en France. Dans la course au gigantisme et à l'ostentation architecturale du début des années 1930, le " grand " Rex

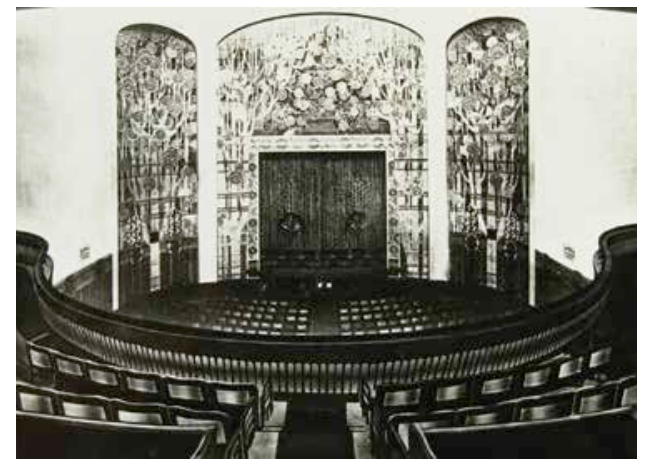

Fig. 13 :

Gambetta Palace,

Paris 20 arrond., Henri

Sauvage arch., 1920.

Vue de l'arcade Art

déco encadrant la scène depuis le second balcon (Source : Vergnes, 1925).

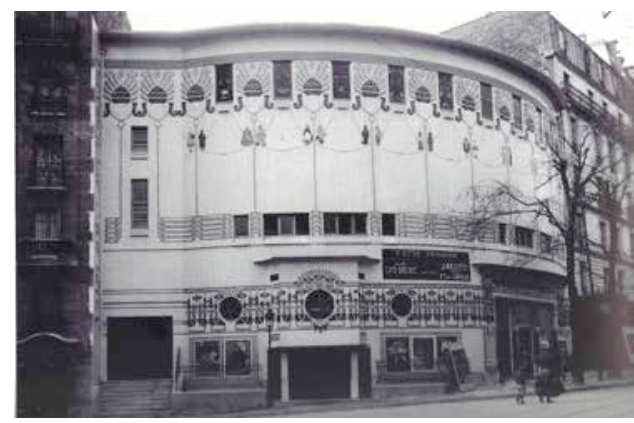

Fig. 14 :

Gambetta Palace, Paris 20 arrond., XXe, façade aux motifs Art déco (Source : Vergnes, 1925).

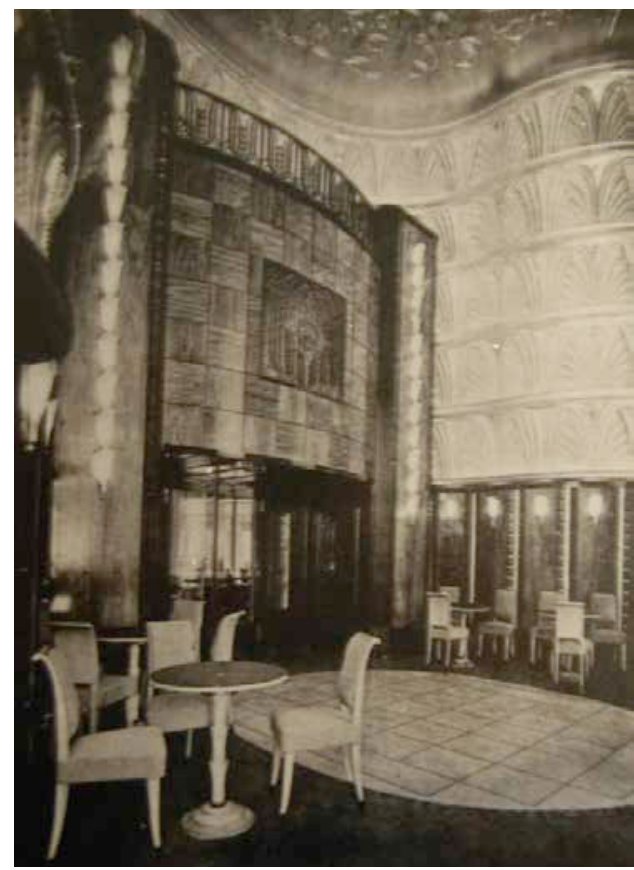

Fig. 15 :

Paramount Palace, Paris 9 arrond., Bluysen \& Verity arch., 1926. Foyer et bar du cinéma dans le style Art déco (d'après les Archives de l'association Eldorado). clôture en beauté le chapitre qu'avait ouvert le Louxor. Le projet de cette immense salle de 3500 places est entrepris, en 1931, par Jacques Haïk, un homme d'affaires plein d'audace, fondateur des établissements éponymes de production de films. 11 est également propriétaire et exploitant de deux grands cinémas à Paris, l'Olympia et le Colisée. Pour la conception de sa nouvelle salle sur les Grands Boulevards, il fait appel à Auguste Bluysen, auteur du Paramount Palace et du théâtre de la Michodière, deux beaux exemples de 
l'Art déco parisien au milieu des années 1920. Cet architecte des édifices de loisirs a fait également preuve d'habileté dans d'autres registres esthétiques en province, notamment dans le style balnéaire avec le casino de Bagnoles-de-l'Orne et l'hôtel Westminster au Touquet-Paris-Plage (Klein, 1994). Le commanditaire du Rex lui adjoint, néanmoins, le décorateur Maurice Dufrêne, connu pour son design de mobilier, ses modèles dans les arts décoratifs et en particulier son travail, depuis 1921, aux Galeries Lafayette (Samoyault-Verlet, 2016). Jacques Haïk complète, enfin, son équipe de concepteurs en sollicitant l'ingénieur américain John Eberson, célèbre à cette époque pour les nombreux cinémas qu'il a construits au cours des années 1920, à travers les États-Unis.

D’origine autrichienne, Eberson a été formé à l'université de Vienne avant d'émigrer en Amérique au tout début du siècle (Valentine, 1994 : 38). Aux alentours de 1910, il s'était associé à un

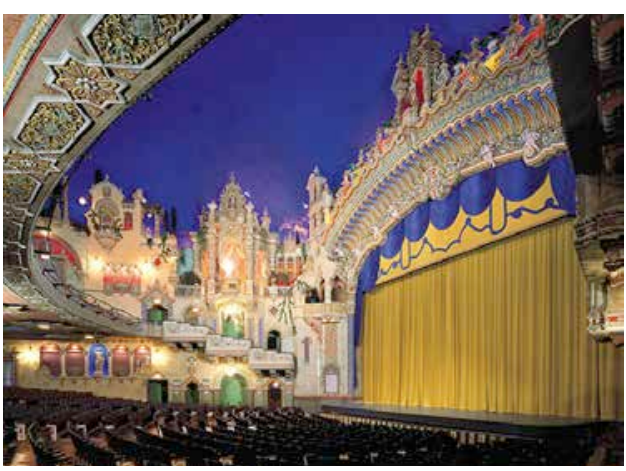

promoteur de théâtres provinciaux, et ensemble ils parcouraient les États-Unis à la recherche de villes ou de villages qui voudraient bien s'équiper d'un Opera House. lls rencontrèrent alors un grand succès ce qui lui a valu le surnom de Opera House John (Hall, 1988 : 95). Cependant, ses premières réalisations d'envergure datent du début des années 1920, comme le Majestic Theatre de Houston (1922) (fig. 16). Dans ce projet, il expérimente un concept qui lui rapportera de nombreuses commandes et fera école en matière d'architecture de cinéma aux États-Unis. Son idée est simple, loin d'être sans précédent, et consiste à édifier le cinéma comme " un magnifique amphithéâtre, sous le ciel nocturne, pleine lune, dans un jardin italien, une cour royale persane, un patio espagnol, ou encore dans la cour d'un mystérieux temple égyptien. " (Hall, 1988 : 96). Il s'agit donc de créer par un décor exotique l'illusion d'une salle en plein air, d'où, d'ailleurs, le qualificatif d'" atmosphérique "pour ce type de salle dont il se fera le promoteur.

Tout au long des années 1920, Eberson exploite et développe ce concept jusqu'à l'excès, dessinant des dizaines de projets majoritairement réalisés. Or, l'élan de la construction des salles atmosphériques est déjà en passe de décliner en Amérique, lorsque Jacques Haïk fait venir Eberson à Paris pour l'associer à Dufrêne et Bluysen. De la collaboration de ces quatre personnages, un homme d'affaires audacieux, un ingénieur soi-disant architecte, un décorateur plutôt desi-

Fig. 17 :

Rex, Paris 2 arrond.,

Bluysen \& Eberson arch. et Dufrêne décorateur, 1932. Décor

" atmosphérique " de la salle conçu vraisemblablement par

Eberson (Source : le site de l'établissement : www.legrandrex.com).

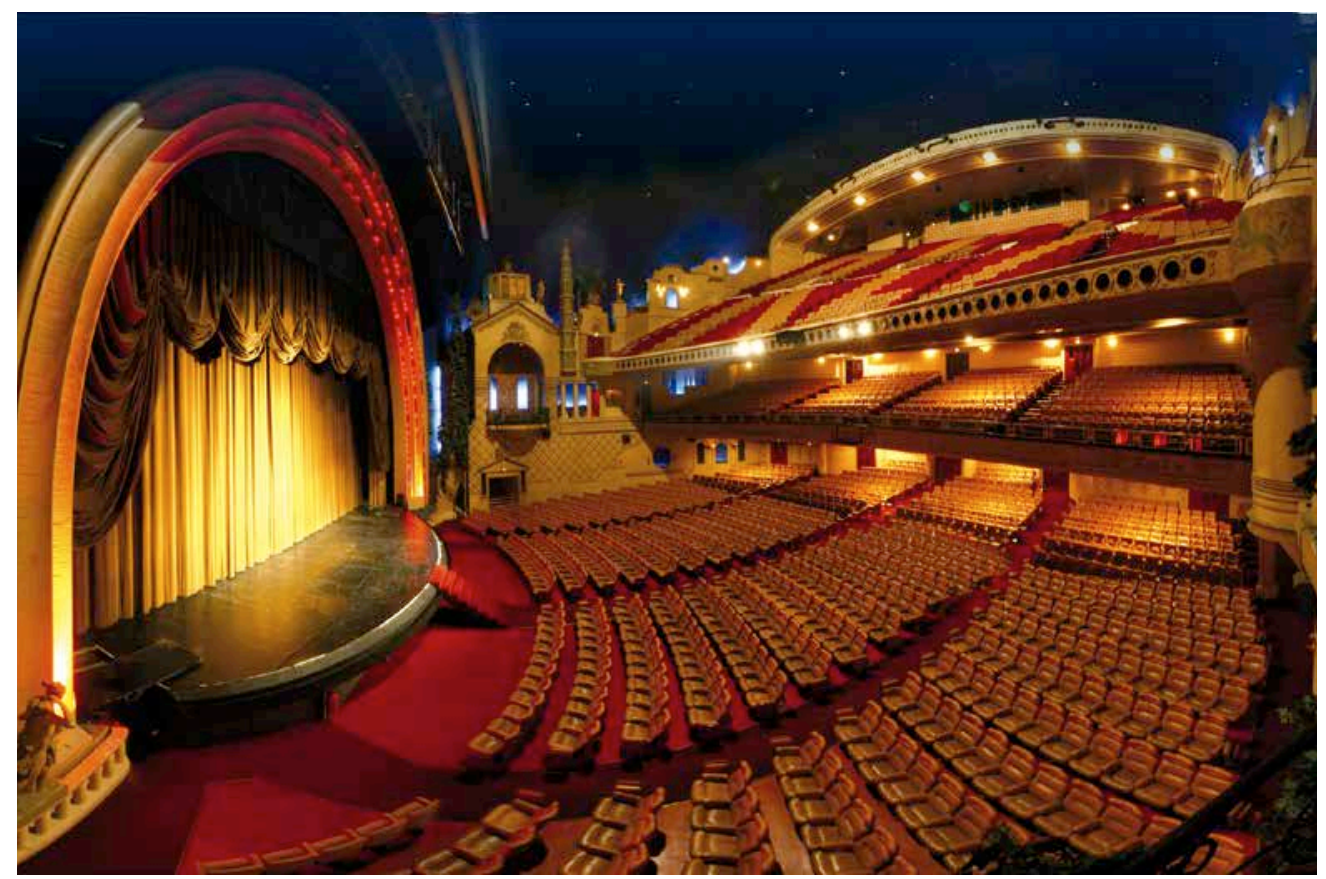


gner, et un architecte également décorateur, résulte une œuvre hybride, mélangeant l'exotisme à l'Art déco et au modernisme. La salle frappe d'abord par son décor " atmosphérique ", signature d'Eberson qui s'est inspiré, pour le Rex, d'un village mauresque, voire maghrébin avec toutefois quelques éléments classicisants, l'ensemble recouvert d'un plafond bleu de ciel nocturne, criblé de constellations d'ampoules électriques (fig. 17). L'ampleur et la décoration des espaces d'accueil attirent ensuite l'attention, de nombreux foyersbars spacieux répartis sur les trois niveaux et décorés dans un style Art déco, portent la touche de Maurice Dufrêne (fig. 18). Enfin, de l'extérieur, l'édifice impressionne par son aspect imposant, en proue de navire sur l'angle de la rue et du boulevard Poissonnière, rappelant le style " paquebot " des modernistes par une façade aveugle et dépouillée, couronnée seulement d'une tourelle lumineuse portant l'enseigne REX (fig. 19). Si l'on suppose une répartition des tâches entre les protagonistes du projet, cette composition de façade sans rapport aucun avec l'exotisme de la salle, est fort probablement l'apport de Bluysen.

Après l'examen de ces trois exemples d'exotisme dans les cinémas de Paris, on peut se demander comment ces œuvres insolites sont reçues dans le milieu professionnel et chez les Parisiens. Le Louxor fait partie des premiers cinémas publiés dans les revues d'architecture. C'est La Construction moderne l'un des périodiques spécialisés les plus importants, mais aussi l'un des premiers à s'intéresser à l'architecture cinématographique qui reproduit la façade et une photographie intérieure de la salle égyptisante dès sa construction, mais sans commentaire ni explication ${ }^{6}$. Or, malgré l'inauguration réussie de la salle, le changement immédiat de propriétaire à la suite de la faillite et la mort d'Henri Silberberg, puis les travaux entrepris par le nouveau propriétaire, la compagnie Pathé, en 1930, le décor égyptien tombera au bout d'une décennie dans l'oubli, et bientôt recouvert même sous des couches de plâtre et de peinture, jusqu'à la redécouverte de ce patrimoine cinématographique et architectural à la fin du siècle, puis sa restauration à l'identique sous la pression et grâce aux efforts des riverains cinéphiles. Quant au cinéma Sèvres, sa publication dans le recueil édité par Eugène Vergnes au milieu des années 1920 ne pourra pas empêcher sa disparition dans les années 1960. Parmi les trois salles évoquées, le Rex aura connu la fortune critique la plus intéressante. Peu

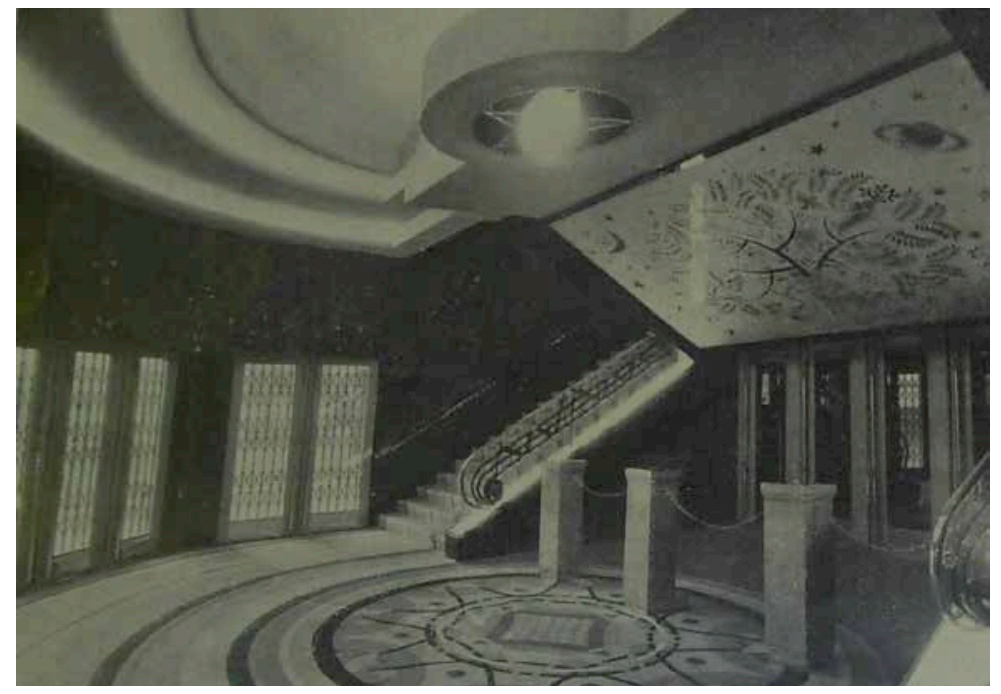

après son ouverture, L'Architecture d'aujourd'hui, une revue influente d'avant-garde architecturale, lui réserve un accueil plutôt acerbe : "Avec le Rex, nous sommes transportés en Amérique, écrit Pierre Vago, le rédacteur de la revue, M. Bluysen assisté de l'Anglo-Saxon Eberson, nous a donné la première (et, nous l'espérons, la dernière) salle atmosphérique [...] Que de telles idées saugrenues, poursuit-il, aient du succès en Amérique, nous le comprenons encore ; mais pour que le Rex n'ait pas été plus fraîchement accueilli par le public parisien, il a fallu le long abrutissement du public par le film et le music-hall "(Vago, 1932 : 31). Le chroniqueur oppose, implicitement, à l'aberration du Rex, la " simplicité harmonieuse " du Gaumont Palace, le plus grand cinéma d'Europe avec ses 6000 places, construit par l'architecte Henri Belloc. Le contraste esquissé entre le Rex et le Gaumont Palace n'est guère anodin. Ces cinémas représentent, en effet, les exemples extrêmes des tendances décoratives des années 1930. Si le premier incarne la salle Art déco aux accents exotiques tardifs, le second manifeste un mode de décor aspirant aux idées du Mouvement moderne, recherché dans le tracé même des lignes et des volumes de la salle, souligné par l'éclairage (fig. 20) ; d'où le penchant évident de l'Architecture d'aujourd'hui pour le Gaumont Palace. Dans La Construction moderne, en revanche, Antony Goissaud fait l'éloge du Rex, le qualifiant de " l'œuvre prodigieuse entreprise par Jacques Haïk ", félicitant ses réalisateurs " l'architecte Auguste Bluysen au talent incontesté et le maître décorateur Maurice Dufrêne dont la réputation n'est plus à faire ", sans toutefois citer l'Américain Eberson, pourtant le père des salles atmosphériques (Goissaud, 1933 : 247).
Fig. 18 :

Rex, Paris 2 arrond., décoration de la rotonde d'entrée, l'œuvre de Maurice Dufrêne (Source

: La Construction moderne, 48e année, $n^{\circ} 16$, p. 242).

6. La Construction moderne, 37 année, $n^{\circ} 26$. 

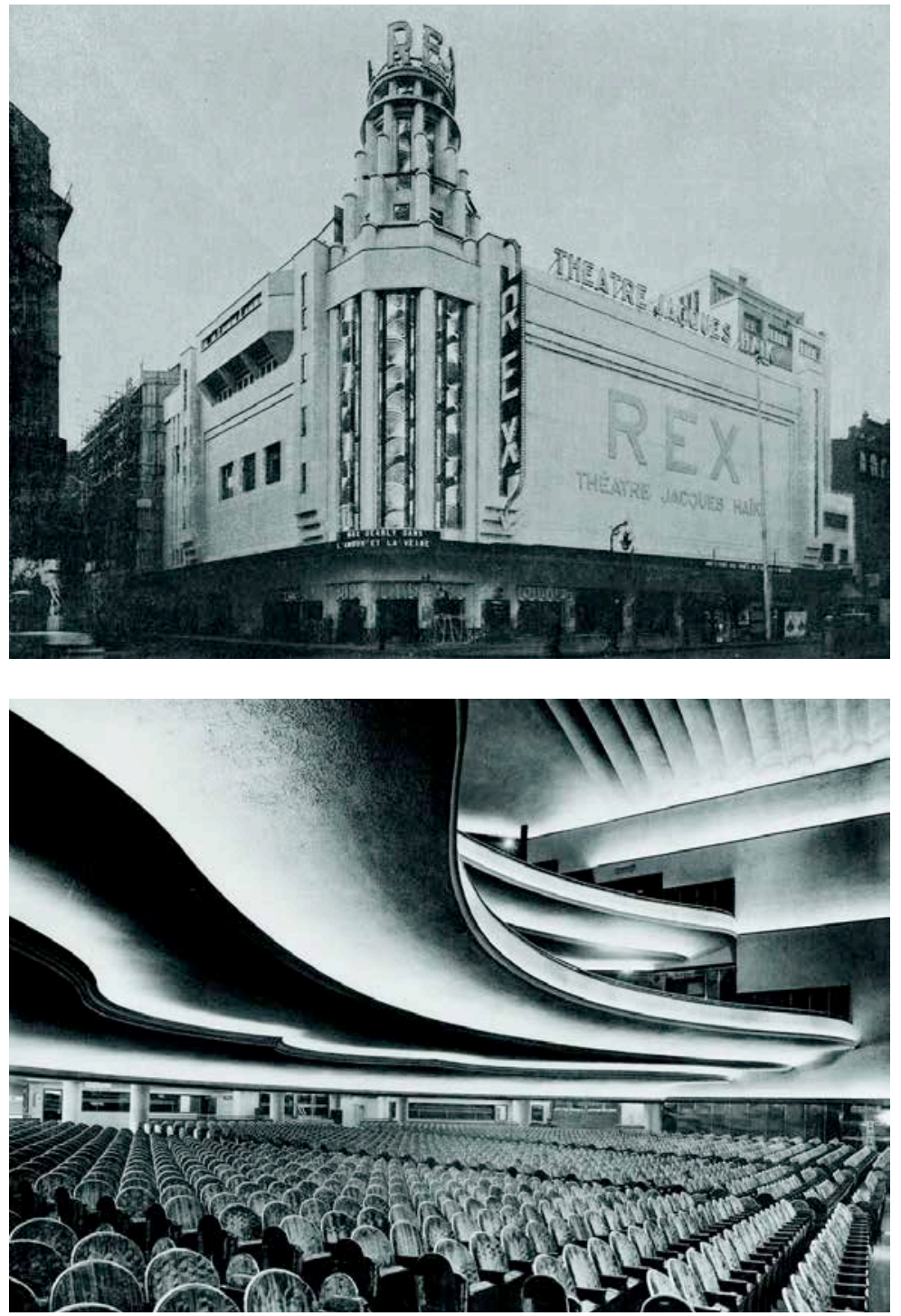

Fig. 19 : Rex, Paris 2 arrond., façade conçue proche $d u$ style paquebot, probablement par Auguste Bluysen (Source

: La Construction moderne, 48e année $\left.n^{\circ} 16, p l .61\right)$.

Fig. 20 : Gaumont Palace, Paris 18 arrond., Henri Belloc arch., 1930. Exemple de décor moderniste consistant dans le jeu des formes et l'éclairage (Source : L'Architecte, Décembre 1931, Pl. 70).
Cette réception controversée du Rex se reflète dans sa destinée : d'une part, conformément au vœu de Pierre Vago, il demeure la première et la dernière salle atmosphérique de Paris ; d'autre part, son exotisme extravagant semble plaire aux Parisiens car malgré la faillite de Jacques Haïk quelques années plus tard, il deviendra l'une des salles mythiques, théâtre des évènements les plus mondains de Paris (Lacloche, 1981 : 97). Au début des années 1980, alors que le Gaumont Palace, l'antithèse esthétique des salles exotiques, aura été démoli depuis une décennie, le Rex et le Louxor seront inscrits à l'Inventaire supplémentaire des monuments historiques. Nul doute que les décors insolites et exotiques de ces salles, ces " idées saugrenues " selon le critique d'architecture moderniste, ayant fait rêver des générations de spectateurs, ont été le principal garant de la survie de ces cinémas d'antan.

\section{Références}

Abadie, S. (2018). Architecture des salles obscures. Paris 1907-1939. Paris : AFRHC.

Abadie, S. (2012). Une histoire architecturale de cinémas, genèse et métamorphoses de l'architecture cinématographique à Paris (1907-1939). Thèse de doctorat, Strasbourg: Université de Strasbourg.

Delaire, E. (1907/2004). Architectes élèves de l'école des Beaux arts, 1793-1907. Villierssur-Marne: Phénix Éditions.

Epron, J.-P. (éd.). (1992). Architecture une anthologie, tome 2, les architectes et le projet. Liège: Pierre Mardaga.

Epron, J.-P. (1997). Comprendre l'éclectisme. Paris: Norma.

Goissaud, A. (1933). Le Rex, Cinéma-Théâtre à Paris. La Construction moderne, 48(16), 238-247.

Hall, B. M. (1961/1988). The best remaining seats : the golden age of the movie palace. NewYork: Da Capo Press.

Heathcote, E. (2001). Cinema Builders. Londres: Wiley-Academy.

Humbert, J.-M. \&t Pumain, Ph. (éd.). (2013). Le Louxor, palais du cinéma. Bruxelles: Archives d'architecture moderne.

Humbert, J.-M. (2010). Le palais de l'Égypte à l'Exposition universelle de 1900. In H. Morlier, (éd.), L'architecte Marcel Dourgnon et l'Égypte. Paris : INHA. Récupéré de http:// inha.revues.org/7008

Humbert, J.-M, Pantazzi, M. \&t Ziegler, Ch. (1994). Égyptomanie, l'Égypte dans l'art occidental (1730-1930). Paris/Ottawa: Réunion des musées nationaux/Musée des beaux-arts du Canada.

Kinsilla, E. B. (1917). Modern Theatre Construction. New-York: The Moving Picture Wolrd.

Klein, R. (1994). La côte d'Opale des années trente, Le Touquet Paris-Plage. Paris: Norma.

Lacloche, F. (1981). Architectures de cinémas. Paris: Moniteur.

Meusy, J.-J. (2002). Paris-Palaces ou le temps des cinémas (1894-1918). Paris: CNRS.

Minnaert, J.-B. (2002). Henri Sauvage ou l'exercice du renouvellement. Paris: Norma.

Oulebsir, N. \&t Volait, M. (éd.). (2009). L'orientalisme architectural, entre images et savoirs. Paris : CNRS/Picard. 
Peltre, Ch. (2004/2010). Orientalisme. Paris: Terrail.

Russell, F. (éd.). (1982). L'architecture de l'Art nouveau, traduit de l'anglais par Florence Sébastiani. Paris: Berger-Levrault.

Samoyault-Verlet, C. (2016). Dufrêne Maurice (1876-1955). In Encyclopædia Universalis. Récupéré de http://www.universalis.fr/ encyclopedie/maurice-dufrene/
Vago, P. (1933). Les grands cinémas parisiens. Architecture d'aujourd'hui, 4(7), 31.

Valentine, M. (1994). The show starts on the side walk: an architectural history of the movie theatre, starring S. Charles Lee. New Haven/ London: Yale University Press.

Vergnes, E. (1925). Cinémas, vues extérieures et intérieures, détails, plans. Paris: Ch. Massin. 